\title{
Retrospective Study of Carfilzomib-Pomalidomide- Dexamethasone in Relapsed/Refractory Multiple Myeloma Patients in a Tertiary Care Hospital in India
}

\author{
Pallavi Mehta $^{1} \cdot$ Neha Yadav $^{1} \cdot$ Bhaarat Folbs $^{1} \cdot$ Jyotsna Kapoor $^{1} \cdot$ \\ Vishvdeep Khushoo $^{1} \cdot$ Narendra Agrawal $^{1} \cdot$ Rayaz Ahmed $^{1} \cdot$ Dinesh Bhurani $^{1}$
}

Received: 14 January 2021/ Accepted: 15 June 2021 / Published online: 3 July 2021

(C) Indian Society of Hematology and Blood Transfusion 2021

\begin{abstract}
Carfilzomib is a second-in class Proteosome Inhibitor and has been approved for Relapsed/Refractory Multiple Myeloma (RRMM). We retrospectively retrieved and analyzed data of KPd combination both biweekly and weekly regimens at our centre from 1 st August 2017 and 31 st May 2020. Sixty-nine patients were treated with KPd with median age of 58 years. Median prior lines of chemotherapy were 2(1-15). Twenty-eight (40.5\%) patients underwent autoSCT. Median no. of cycles was 4(1-12) and $3(1-13)$ with median time to response of 4(2-12) and 2(2-6) months in biweekly and once weekly regimen cohorts respectively. At last follow-up, overall response rate (ORR) was $65.2 \%$ \{CR-n $=10(14.5 \%)$, VGPR-n $=19(27.5 \%)$, PR-n $=16(23.2 \%)\}$ with $\mathrm{n}=13(18.8 \%)$ patients had PD and relapse was observed in $n=24(34.8 \%)$. Thirty (43.4\%) patients received maintenance therapy $\{\mathrm{n}=21(70 \%)\}$ or autoSCT $\{\mathrm{n}=9(30 \%)\}$. Common toxicities were anemia $\{\mathrm{n}=11(15.9 \%)\}$, thrombocytopenia $(\mathrm{n}=15(21.7 \%)$ and neutropenia $(\mathrm{n}=16(23.2 \%)\}$, hypertension $\{\mathrm{n}=$ $28(40.5 \%)\}$, peripheral neuropathy (grade1/2) $\{\mathrm{n}=$ $15(21.7 \%)\}$, infections $[\mathrm{n}=18(26 \%)$ bacterial $[\mathrm{n}=$ $9(13 \%)$, viral $n=7(10.1 \%)$, fungal $n=8(11.6 \%)\}]$. At a median follow-up of 18 months, the estimated median PFS was 11.3 months (95\%C.I. 8.3- 14.2) whereas the estimated median OS was 28 months (95\%C.I. 20.4-35.5) for the entire cohort. Mortality rate of $2.5 \%$ and $10 \%$ in two cohorts respectively. Commonest cause of death was PD and sepsis. KPD is a well-tolerated regimen for RRMM,
\end{abstract}

Pallavi Mehta

dr_pallavimehta@yahoo.in

1 Rajiv Gandhi Cancer Institute and Research Centre, Sector-5, Rohini, Delhi 110085, India which can be a bridge to ASCT, however with significant side effects.

Keywords Refractory myeloma - Carfilzomib .

Autologous transplant

\section{Introduction}

Multiple myeloma (MM) is a plasma cell dyscrasia which has relished the emergence of various novel agents in the last few decades. Unfortunately, relapses are still inevitable part of natural course of this debilitating disorder. At each relapse, treatment choice becomes a complex decision. Various clinical trials have demonstrated the efficacy of combination of proteasome inhibitors (PIs), immunomodulatory drugs (IMiDs) and dexamethasone; in newly diagnosed as well as Relapse/Refractory MM (RRMM) patients. However a large number of these patients become refractory to bortezomib or lenalidomide on relapses and pose immense therapeutic challenge [1].

Over last few decades, newly approved antimyeloma agents have shown promising effect. Carfilzomib, secondin class irreversible PI, has been approved for patients who are refractory to minimum 2 lines of prior therapies; including bortezomib and IMiDs or who showed progression of disease while on chemotherapy [2].

Another agent in armamentarium is pomalidomide which was approved in 2013 in patients who were refractory to atleast 2 lines of chemotherapy [3, 4].

Preclinical and clinical data of carfilzomib-pomalidomide-dexamethasone (KPd) combinations support synergistic effect [5-7]. We evaluated this novel combination of KPd in RRMM patients at our centre and hereby, present our initial experience with this novel regimen. The primary 
objective of our study was to assess safety and efficacy. The secondary objective of our study was to study progression free survival (PFS) and overall survival (OS).

\section{Methodology}

\section{Patient Selection}

Records of RRMM patients who had atleast 1 prior anti myeloma therapy (IMiDs/PIs/refractory to either one or both) and received Carfilzomib based regimen at our center between the period of 1-August-2017 and 31-October2018 in biweekly regimen and weekly regimen from 1-November-2018 to 31-May-2020 were reviewed retrospectively. All patients were followed up till 15-August2020. The study was approved by Institutional Review Board.

\section{Study Design}

This is a retrospective study where medical records of all eligible patients were reviewed including demographics, baseline characteristics \{complete blood count, calcium, creatinine, Lactate dehydrogenase, bone marrow aspiration/biopsy, cytogenetics or Fluorescence-In-situ hybridization (FISH), skeletal evaluation, immune profile, serum free light chain assays, immunofixation electrophoresis, serum M-protein Electrophoresis\}, International System of Staging (ISS) or Revised International System of Staging (R-ISS), prior lines of chemotherapy, responses, KPd toxicity profile, post KPd disease status and maintenance therapy. Baseline ECG was done in all patients and ECHO was done in patients with comorbidities. Data on autologous stem cell transplantation (AutoSCT) pre and post KPd was also collected.

\section{KPd Protocol}

\section{Biweekly regimen}

Intravenous Carfilzomib was given on day $1-2,8-9$ and $15-16$ of each cycle. The dose of Carfilzomib @20mg/m on day 1-2 and thereafter @ $27 \mathrm{mg} / \mathrm{m}^{2}$ from week 2 (cycle1) and @27mg/m from cycle 2 onwards) was administered.

\section{Once weekly regimen}

Intravenous Carfilzomib was given on day 1 (day 1-2 in cycle 1 only ), day 8 and day 15 of each cycle @ 20mg/m² on day 1 and day 2 followed by $36 \mathrm{mg} / \mathrm{m}^{2}$ in weekly doses with dose modification as per tolerability).
Pomalidomide $4 \mathrm{mg}$ on days 1-21 and dexamethasone $20 \mathrm{mg} / \mathrm{IV}$ weekly in 28 days treatment cycles [6]. Carfilzomib was withheld or dose reduction was done in patients with grade-3/4 hematological or non-hematological toxicities in both regimen. Pomalidomide dose adjustment was also done according to grade-3/4 hematological and nonhematological toxicities.

After response assessment patients were offered for AutoSCT (if willing). Patients not willing for autoSCT received maintenance treatment, among which who opted for carfilzomib/pomalidomide maintenance therapy received $36 \mathrm{mg} / \mathrm{m}^{2}$ of carfilzomib on day-1 and 15 and pomalidomide $4 \mathrm{mg}$ on day $21 / 28$ until progression or intolerance [8]. Rest received alternate maintenance therapy. All patients were followed up until last follow-up visit or death.

\section{Efficacy Assessment}

Responses were assessed as per International Myeloma Working Group (IMWG) uniform response criteria [9]. Interim assessment was done after every 2 cycles. Primary efficacy endpoints were overall response rate (ORR) i.e. percentage of patients who achieved stringent complete response(sCR), complete response(CR), very good partial response(VGPR), partial response(PR) using IMWG response criteria [9] at last follow-up. Relapse is defined as per IMWG uniform response criteria [9]. Progressive disease (PD) is defined as per IMWG uniform response criteria [9]. Secondary efficacy endpoints were duration of response (time from first confirmed response to progressive disease (PD)/death/PFS/last follow-up visit). Patients must have completed atleast 2 cycles of KPd therapy for efficacy evaluation. Refractoriness to bortezomib and lenalidomide is defined if patients progressed while receiving bortezomib or lenalidomide or both containing regimens.

\section{Safety Assessment}

Both hematological and non-hematological toxicities were recorded till last follow-up. Criteria for hematological toxicities were defined as anemia $\{$ hemoglobin $(\mathrm{Hb}):<8 \mathrm{gm} /$ $\mathrm{dl}$ \}, Grade-3/4 neutropenia \{absolute neutrophil coun$\mathrm{t}(\mathrm{ANC}):<1000 / \mathrm{cumm}$, grade-3/4 thrombocytopenia \{platelet counts: $<20,000 /$ cumm $\}$. Non-hematological toxicities recorded were incidence of infections, cardiac toxicities or changes in ECG/ECHO after every 2-4 cycles as well as hypertension and peripheral neuropathy.

\section{Statistical Analysis}

Statistical analyses were conducted for patients who received at least 2 cycles of KPd therapy. Baseline 
demographics and clinical characteristics were summarized using descriptive statistics. Toxicities were summarized by using Adverse Event(AE) grading [10]. Time to response was defined as from start of treatment to first documentation of response of either PR or better and was calculated from first administration of KPd therapy and best response achieved. The duration between initiation of KPd treatment and PD, according to specified criteria, was defined as the PFS of MM. OS was defined as the duration from start of KPd therapy until death or date of last follow-up. Time to event outcome such as PFS and OS were estimated by using Kaplan-Meier curves along with estimates of median and $95 \% \mathrm{CI}$. The statistical analysis was performed using SPSS version 21.0 of IBM.

\section{Results}

\section{Baseline Demographics}

Total 69 patients were treated with KPd regimen during study period. Median age was 58 (32-74 years) with male ratio of $57.9 \%(\mathrm{n}=40)$. Most common co-morbidities were hypertension $(n=20)$ followed by diabetes mellitus $(n=14)$. At baseline presentation, bone disease $\{n=43(62.3 \%)\}$ was the most common presenting complaint. Most common ISS staging at diagnosis was ISS-III $\{\mathrm{n}=28(40.5 \%)\}$ and subtype was IgG myeloma $\{\mathrm{n}=26(37.6 \%)\}$ followed by Light chain myeloma $\{\mathrm{n}=20(28.9 \%)\}$ and $\operatorname{IgA}$ myeloma $\{\mathrm{n}=$ $6(8.7 \%)\}$. FISH was available in $\mathrm{n}=25(36.2 \%)$ patients \{positive for del13q( $\mathrm{n}=1) ; \operatorname{del} 17 \mathrm{p}(\mathrm{n}=2) ; \mathrm{t}(11 ; 14)(\mathrm{n}=1) ; 1 \mathrm{q}$ gain $(\mathrm{n}=1), 1 \mathrm{p} \operatorname{del}(\mathrm{n}=1)$; Del 13q and 1q gain $(\mathrm{n}=1)$; Del $13 q /$ del $17 \mathrm{p} / \mathrm{t}(4,14)(\mathrm{n}=1)$; Del 17p and del 13q $(\mathrm{n}=1)$; Del $17 \mathrm{p}$ and del 13q, t(4,14) $(\mathrm{n}=1)$; Hyperdiplod, del 13q, 1q amp- $(\mathrm{n}=1)$; Normal $(\mathrm{n}=14)\}$. (Table 1).

\section{Pre KPd Therapy}

Median number of prior lines of chemotherapy was 2(1-15). Fifty-eight (84\%) patients were refractory to both bortezomib and lenalidomide; whereas $\mathrm{n}=39(56 \%)$ were to bortezomib and $63.8 \%$ were to lenalidomide. Twentyeight $(40.5 \%)($ biweekly:n $=11$; weekly regimen:n $=17)$ patients underwent autoSCT including $n=3 / 11$ received two SCT (Tandem ASCT $=2$; ASCT and Allo-SCT $=1$ ). $\mathrm{N}$ $=34(49.2 \%)$ had relapse whereas $\mathrm{n}=28(40.5 \%)$ were primary refractory and $n=7(10.1 \%)$ had SD. (Table 1 ).

\section{Response to KPd Regimen}

Median number of KPd cycles in biweekly and weekly regimen were 4(1-12) and 3(1-13) respectively. Median number of KPd cycles after which response assessment was done was 4(2-12) and 2(2-13) cycles and median time to treatment response was 4(2-12) months and 2(2-6) months in these regimen respectively.

Post 2 cycles, responses were $\operatorname{CR}(n=3), \operatorname{VGPR}(n=17)$, $\mathrm{PR}(\mathrm{n}=27)$. Ten patients had PD, whereas 5 had SD and $n$ $=7$ were not assessed (lost to follow-up $=5$; death $=1$, not available $=1$ ).

At last follow-up, ORR was $65.2 \%\{\mathrm{CR}-\mathrm{n}=10(14.5 \%)$, VGPR-n $=19(27.5 \%)$, PR-n $=16(23.2 \%)\} . \mathrm{N}=$ $13(18.8 \%)$ patients had PD whereas $n=4(5.8 \%)$ had SD. Six $(8.7 \%)$ patients were lost to follow-up and death $=1$ $(1.4 \%)$. Relapse was observed in $n=24(34.8 \%)$. (Table 1)

\section{Toxicities and Infection}

\section{Hematological Toxicities}

Hematological toxicities such as anemia was seen in $11(15.9 \%)$ patients, thrombocytopenia in $15(21.7 \%)$ patients $\{$ grade- $3 / 4=66.6 \% ; n=10 / 15\}$ and neutropenia in $16(23.2 \%)$ patients $\{$ grade- $3 / 4=25 \% ; n=4 / 16\}$. Differences in both regimens has been shown in Table 2 .

\section{Non-hematological toxicities}

Non haematological toxicities such as cardiac toxicities and peripheral neuropathy were recorded. Carfilzomib induced hypertension was seen in $28(40.5 \%)$ patients, out of which $13(18.8 \%)$ patients had prior history of hypertension. Peripheral neuropathy(grade-1/2) was seen in $15(21.7 \%)$ patients which was diagnosed on the basis of clinical history. One patient had Carfilzomib induced hyponatremia. Congestive heart failure (CHF) was seen in $2(2.8 \%)$ patients which was diagnosed on the basis of clinical examination and echocardiography findings. Differences in both regimens has been shown in Table 2 .

\section{Infections}

Febrile episodes were seen in 18 (26\%) patients out of which 9 had documented bacterial infection (gram positive $=2$; gram negative $=7$ ) (Table 2) .

The common site for bacterial isolation was urinary tract $(n=4 / 9)$ followed by bloodstream $(n=5 / 9)$. Seven $(10.1 \%)$ patients had documented respiratory viral infections $\{(\mathrm{H} 1 \mathrm{~N} 1:(\mathrm{n}=5)$; Para-influenza: $(\mathrm{n}=1) \&$ rhinovirus and coronavirus: $(\mathrm{n}=1)\}$.

Eight $(11.6 \%)$ patients had possible invasive fungal infection(IFI) including 2(2.8\%) patients with candidiasis. Cryptosporidium parvum was seen in $1(1.4 \%)$ patient (Table 2). Upper respiratory tract infection of unknown aetiology was observed in 11(15.9\%) patients. One (1.4\%) patient had acute serous otitis media (Table 2). 
Table 1 Baseline Patient demographics, disease and treatment characteristics and Response Post KPd

\begin{tabular}{|c|c|c|}
\hline Variables & Biweekly (N) (\%) & Weekly (N) (\%) \\
\hline No. of patients & 39 & 30 \\
\hline Median age at diagnosis & 56 (32-74 years) & $58(36-74$ years $)$ \\
\hline HTN & $13(33.3 \%)$ & $07(23.3)$ \\
\hline DMT2 & $10(25.6 \%)$ & $04(13.3)$ \\
\hline CAD & None & $03(10)$ \\
\hline CKD & None & $02(6.6)$ \\
\hline \multicolumn{3}{|l|}{ Sex } \\
\hline Males & $20(51.2)$ & $20(66.6)$ \\
\hline Females & $19(49.8)$ & $10(33.4)$ \\
\hline \multicolumn{3}{|l|}{ ISS at diagnosis } \\
\hline ISS-1 & $05(12.8)$ & 08 (26.6) \\
\hline ISS-2 & $08(20.5)$ & $09(30)$ \\
\hline ISS-3 & $18(46.1)$ & $10(33.3)$ \\
\hline NA & $08(20.5)$ & $03(10)$ \\
\hline FISH available & $10(25.6)$ & $15(50)$ \\
\hline Del13q & 01-Oct & None \\
\hline Del 17p & 01-Oct & Jan-15 \\
\hline $\mathrm{t}(11 ; 14)$ & 01-Oct & None \\
\hline 1q gain & None & Jan-15 \\
\hline $1 \mathrm{p}$ del & None & Jan-15 \\
\hline Del $13 \mathrm{q}$ and $1 \mathrm{q}$ gain & None & Jan-15 \\
\hline Del13q/del17p/t $(4,14)$ & None & Jan-15 \\
\hline Del $17 p$ and del $13 q$ & None & Jan-15 \\
\hline Del $17 \mathrm{p}$ and del $13 \mathrm{q}, \mathrm{t}(4,14)$ & None & Jan-15 \\
\hline Hyperdiplod, del 13q,1q amp & None & Jan-15 \\
\hline Negative & 07-Oct & Jul-15 \\
\hline Median number of Prior regimens & 3 (1-15 lines) & 02 (1-5 lines) \\
\hline \multicolumn{3}{|l|}{ Prior Therapies } \\
\hline Stem Cell Transplantation & $11(30.5)$ & $17(56.6)$ \\
\hline Lenalidomide & None & $08(26.6)$ \\
\hline Bortezomib & $4(10.3)$ & None \\
\hline Bortezomib + Lenalidomide & 35 (89.7) & $22(73.3)$ \\
\hline \multicolumn{3}{|l|}{ Pre KPd Response } \\
\hline Primary Refractory & $25(64.1)$ & $03(10)$ \\
\hline Relapse & $10(25)$ & $24(80)$ \\
\hline SD & $04(11.1)$ & $03(10)$ \\
\hline \multicolumn{3}{|l|}{ Response Post KPd Post 2 cycles } \\
\hline $\mathrm{CR}$ & $03(7.7)$ & None \\
\hline VGPR & $08(20.5)$ & $09(30)$ \\
\hline PR & $09(23.1)$ & $18(60)$ \\
\hline SD & $02(5.1)$ & $02(6.6)$ \\
\hline PD & $09(23.1)$ & $01(3.4)$ \\
\hline No response & $01(2.6)$ & None \\
\hline Assessment not done & $06(15.4)$ & None \\
\hline Lost to follow -up & 05-Jun & None \\
\hline Death & 01-Jun & None \\
\hline Not Available & $01(2.6)$ & None \\
\hline
\end{tabular}


Table 1 continued

\begin{tabular}{lll}
\hline Variables & Biweekly $(\mathrm{N})(\%)$ & Weekly (N) (\%) \\
\hline Response at last follow-up & & \\
ORR & $20(51.2)$ & $25(83.3)$ \\
CR & $08(20.5)$ & $02(6.6)$ \\
VGPR & $04(10.2)$ & $15(50)$ \\
PR & $08(20.5)$ & $08(26.6)$ \\
SD & $02(5.1)$ & $02(6.6)$ \\
PD & $11(28.2)$ & $02(6.6)$ \\
Assessment not done & $06(15.4)$ & $01(3.3)$ \\
Lost to follow -up & $05 / 06$ & 0101 \\
Death & $01 / 06$ & None \\
Relapse & $13(59.1)$ & $11(36.6)$ \\
\hline
\end{tabular}

Table 2 Toxicities and Infections

\begin{tabular}{|c|c|c|}
\hline Variables & $\begin{array}{l}\mathrm{N}(\%) \text { Biweekly } \\
\text { Regimen }\end{array}$ & $\begin{array}{l}\mathrm{N}(\%) \text { Weekly } \\
\text { Regimen }\end{array}$ \\
\hline \multicolumn{3}{|l|}{ Hematological Toxicities } \\
\hline Anemia & $08(20.5)$ & $3(10)$ \\
\hline Thrombocytopenia & $09(23.1)$ & $6(20)$ \\
\hline Grade-3 & 05/09 & None \\
\hline Grade-4 & $04 / 09$ & 01-Jun \\
\hline Neutropenia & $11(28.2)$ & $05(16.6)$ \\
\hline Grade-1/ 2 & $07 / 11$ & 05-May \\
\hline Grade-3/ 4 & $04 / 11$ & None \\
\hline TMA & None & $01(3.3)$ \\
\hline \multicolumn{3}{|l|}{ Non hematological Toxicities } \\
\hline Fatigue & $07(17.9)$ & None \\
\hline Neuropathy (grade-1/2) & $10(25.6)$ & $05(16.6)$ \\
\hline Hypertension & $20(51.3)$ & $08(26.6)$ \\
\hline Cardiac failure & None & $02(6.6)$ \\
\hline Hyponatremia & $01(2.6)$ & None \\
\hline \multicolumn{3}{|l|}{ Infections } \\
\hline Culture positive bacterial Infections & $05(12.8)$ & $04(13.3)$ \\
\hline Gram Positive & $02 / 05$ & None \\
\hline Gram Negative & $03 / 05$ & 04-Apr \\
\hline Viral infections & $04(10.2)$ & $03(10)$ \\
\hline $\mathrm{H} 1 \mathrm{~N} 1$ & $02 / 04$ & $03(10)$ \\
\hline Rhinovirus and Coronavirus & $01 / 04$ & None \\
\hline Parainfluenza & $01 / 04$ & None \\
\hline Possible invasive fungal infections & $06(15.4)$ & $02(6.6)$ \\
\hline Parasitic (Cryptosporodium) & None & $01(3.3)$ \\
\hline Acute serous otitis media & None & $01(3.3)$ \\
\hline $\begin{array}{l}\text { Upper Respiratory Tract Infection (Unknown } \\
\text { Etiology) }\end{array}$ & None & $11(36.6)$ \\
\hline
\end{tabular}

\section{Maintenance Therapy/AutoSCT}

Post KPD, patients were taken for AutoSCT if willing, otherwise were continued with maintenance therapy.
Median number of KPd cycles in transplant recipients and non transplant recipients was $3(2-6)$ and 6 (2-13) cycles respectively. Thirty (43.4\%) patients received maintenance therapy or autoSCT. $\mathrm{N}=21(70 \%)$ patients were on 
Fig 1 a Median PFS of entire cohort, b Median OS of entire cohort
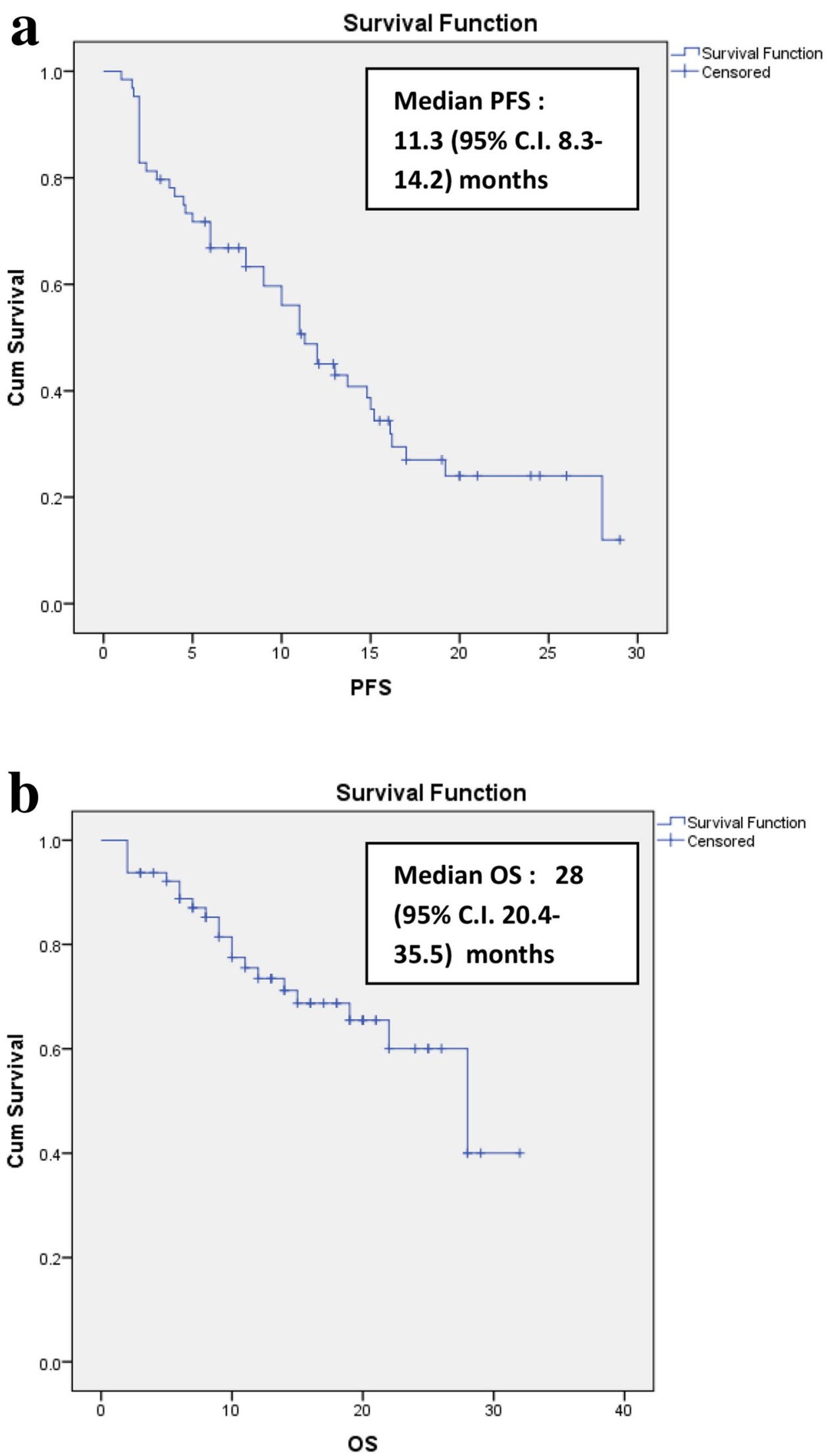
Table 3 Maximum Tolerated dose of Carfilzomib in various studies

\begin{tabular}{lllll}
\hline Study & Median number of lines of therapy & Maximum tolerated dose & \\
\cline { 3 - 5 } & & Carfilzomib & Pomalidomide & Dexamethasone \\
\hline S. Bringhen et al [5] & $8(1-21)$ & $27 \mathrm{mg} / \mathrm{m}^{2}$ (weekly) & $4 \mathrm{mg}$ & $20 \mathrm{mg}$ \\
Shah JJ et al [6] & $6(2-12)$ & $20 / 27 \mathrm{mg} / \mathrm{m}^{2}$ (biweekly) & $4 \mathrm{mg}$ & $40 \mathrm{mg}$ \\
Sonneveld et al [7] & 2 & $20 / 36 \mathrm{mg} / \mathrm{m}^{2}$ (biweekly) & $4 \mathrm{mg}$ & $20 \mathrm{mg}$ \\
Our study (biweekly) & $3(1-15)$ & $20 / 27 \mathrm{mg} / \mathrm{m}^{2}$ (biweekly) & $4 \mathrm{mg}$ & $40 \mathrm{mg}$ \\
Our study (weekly) & $2(1-5)$ & $36 \mathrm{mg} / \mathrm{m}^{2}$ (weekly) & $4 \mathrm{mg}$ & $20 / 40 \mathrm{mg}$ \\
\hline
\end{tabular}

maintenance (carfilzomib-dexamethasone $=5$, pomalidomide-dexamethasone $=9$ and pomalidomide $=8$ ). Nine (30\%) patients received SCT. Median number of days from last day of KPd therapy till day of CD34+stem collection was 23 days (14-42 days) and median CD34+ cell dose was $5 \times 10^{6} / \mathrm{kg}\left(2.83 \times 10^{6} / \mathrm{kg}-7.5 \times 10^{6} / \mathrm{kg}\right)$. Post-SCT response status was $\mathrm{CR}=1$, VGPR $=7$ and $\mathrm{PR}=1$. Of these, 8 received maintenance \{bortezomib/pomalidomide: $(\mathrm{n}=1)$, pomalidomide/dexamethasone: $(\mathrm{n}=5)$, bortezomib: $(\mathrm{n}=1)$, pomalidomide $(\mathrm{n}=1)\}$. One patient received KPd as a consolidation therapy followed by pomalidomide maintenance.

\section{Relapse and Mortality}

Amongst 49 patients who achieved response, relapse rate was $34.7 \%(\mathrm{n}=24)$ whereas $26 \%(\mathrm{n}=18)$ had PD. Mortality rate was $2.5 \%(\mathrm{n}=1)$ and $10 \%(\mathrm{n}=3)$ in biweekly and weekly groups respectively. The commonest cause of death was PD and sepsis.

\section{Survival Outcome}

At a median follow-up of 18 months, the estimated median PFS was 11.3 months (95\%C.I. 8.3-14.2) (Figure 1a) whereas the estimated median OS was 28 months (95\%C.I. 20.4-35.5) for the entire cohort (Figure 1b).

\section{Discussion}

With the emergence of novel agents survival rates have significantly improved for MM patients but relapses still remains one of the important bottleneck. Various combination of PIs with IMiDs agents for myeloma therapy have been studied and proved as an effective therapy.

This retrospective study is conducted look for efficacy and toxicity profile of KPd regimen in a heavily pretreated patient population. Most of the patients (84\%) were refractory to both bortezomib and IMids (Bortezomib only $=56 \%$, IMid only $=63.8 \%$ ). Despite this fact, more than half of our patients responded with ORR of $65.2 \%$ (CR/
VGPR/PR) in biweekly and 90\% (VGPR/ PR) in once weekly regimen after 2 cycles. In studies on biweekly KPD regimen by Shah JJ et al [6] and Sonneveld et al [7], ORR was $50 \%$ and $87 \%$ respectively. For once-weekly KPd S. Bringhen et al [5] reported ORR of $62 \%$. We observed ORR of $83.3 \%$ with $6.6 \%$ :CR, 50\%: $\geq$ VGPR, 26.6\%:PR at last visit. ORR was better in our study probably because of lesser no. of previous lines of therapy and higher Maximum Tolerated Dose (MTD) (carfilzomib @36mg/m2) in weekly regimen. MTD in various studies has been shown in Table 3. Although we observed huge difference in ORR in the two regimens probably due to differences in previous lines of therapies in both groups. Despite good response rates, $34.78 \%$ (biweekly: $\mathrm{n}=13$ and weekly: $\mathrm{n}=11$ ) patients had relapse in our study. PD was seen in $18.8 \%$ (biweekly:n $=11$; weekly:n $=2$ ). In contrast to our result, only 9\% patients had PD in the study by Shah et al [6].

Although KPd is an effective regimen, it comes at the cost of significant toxicities. In our study, grade-4 haematological toxicities were common, whereas non haematological toxicities were uncommon. Hematological toxicities observed were thrombocytopenia $(23.1 \%)$ versus (20\%), neutropenia $(28.2 \%)$ versus $(16.6 \%)$ and anemia $(20.5 \%)$ versus $(10 \%)$ in biweekly and once weekly regimens respectively which is comparable to other studies. In biweekly regimen study by Shah JJ et al [6], hematologic AEs were thrombocytopenia and anemia in $16 \%$ and 59\% of patients respectively. Sonneveld et al [7] also reported grade-3/4 hematological toxicity in $30 \%$ of patients. Similarly in once weekly regimen by S. Bringhen et al [5] haematological AEs occurred in $n=37(79 \%)$. In our study nonhematological AEs were mostly infections and cardiovascular. Other notable non-hematological toxicities were hypertension ( $\mathrm{n}=20$ vs. 8 ), peripheral neuropathy $(\mathrm{n}=10$ vs. 5) and respiratory infections ( $\mathrm{n}=4$ vs. 3 ) respectively. Non-hematologic AEs (Grade $\geq 3$ ) reported by Shah JJ et al [6] included CHF, fluid overload, dyspnea, acute renal failure, peripheral neuropathy and infections. Sonneveld et al [7] had reported cardiovascular events (5\%), respiratory events (5\%), infections (20\%) and neuropathy (3\%) as non-hematologic (grade-3/4) AEs. Similarly S. Bringhen 
Table 4 Comparison with other studies

\begin{tabular}{|c|c|c|c|c|c|c|}
\hline Characteristics & Our Study & Bringhen et al [5] & Shah et al [6] & Sonneveld et al [7] & $\begin{array}{l}\text { PX-171-004 Trial } \\
\text { (Vij R et al) [13] }\end{array}$ & $\begin{array}{l}\text { PX-171-003-A0 } \\
\text { Trial } \\
\text { (Jagannath S } \\
\text { et al) [14] }\end{array}$ \\
\hline Protocol & $\begin{array}{l}\text { Intravenous } \\
\text { Carfilzomib was } \\
\text { given on day 1-2, } \\
7-8 \text { and } 15-16 \text { of } \\
\text { each cycle. The } \\
\text { dose of } \\
\text { Carfilzomib@ } 20 \\
\text { mg/m2 on day } \\
1-2 \text { and thereafter } \\
\text { @ } 27 \mathrm{mg} / \mathrm{m} 2 \text { from } \\
\text { week } 2(\mathrm{cycle}-1) \\
\text { and @ } 27 \mathrm{mg} / \mathrm{m} 2 \\
\text { from cycle } 2 \\
\text { onwards) was } \\
\text { given. } \\
\text { Pomalidomide } \\
\text { @ 4mg orally for } \\
\text { once a day for } \\
21 / 28 \text { days and } \\
\text { Dexamethasone } \\
\text { 20mg/IV weekly }\end{array}$ & $\begin{array}{l}\text { 28-day cycles of } \\
\text { oral } \\
\text { Pomalidomide at } \\
\text { fixed dose of } 4 \\
\text { mg on days } 1-21 \\
\text { (1 week off), } \\
\text { oral or } \\
\text { intravenous (iv) } \\
\text { Dexamethasone } \\
40 \text { mg on days } 1 \text {, } \\
8,15,22 \text { and iv } \\
\text { Carfilzomib at } \\
\text { escalating doses } \\
\text { on days } 1,8,15 . \\
\text { Escalation } \\
\text { started at the } \\
\text { dose of } 36 \text { mg/ } \\
\text { m2 (0 level) and } \\
\text { used a standard } \\
3+3 \text { schema } \\
\text { based on dose- } \\
\text { limiting } \\
\text { toxicities } \\
\text { (DLTs) } \\
\text { occurring in } \\
\text { cycle } 1 . \\
\text { Treatment was } \\
\text { continued until } \\
\text { relapse or } \\
\text { intolerance. }\end{array}$ & $\begin{array}{l}\text { Carfilzomib IV on } \\
\text { days } 1,2,8,9 \text {, } \\
15 \text {, and } 16 \\
\text { (starting dose of } \\
20 / 27 \mathrm{mg} / \mathrm{m}(2) \text { ), } \\
\text { pomalidomide } \\
\text { once daily on } \\
\text { days } 1 \text { to } 21 \text { (4 } \\
\text { mg as the initial } \\
\text { dose level), and } \\
\text { dexamethasone } \\
\text { (40 mg oral or } \\
\text { IV) on days } 1,8, \\
\text { 15, and } 22 \text { of } \\
\text { 28-day cycles. }\end{array}$ & $\begin{array}{l}\text { Intravenous } \\
\text { carfilzomib (20/ } \\
\text { 36mg/m2, days } \\
1,2,8,9,15,16) \\
\text { with } \\
\text { Pomalidomide } \\
\text { (4 mg days 1-21) } \\
\text { and } \\
\text { Dexamethasone } \\
\text { (20mg days } \\
\text { 1,2,8,9,15,16). } \\
\text { Patients who had } \\
\text { not previously } \\
\text { received HDM/ } \\
\text { ASCT, HDM } \\
\text { (200 mg/m2) } \\
\text { was } \\
\text { administered } \\
\text { followed by } \\
\text { autologous stem } \\
\text { cell } \\
\text { transplantation } \\
\text { with stem cells } \\
\text { harvested during } \\
\text { after induction } \\
\text { therapy in the } \\
\text { EMN02 trial. } \\
\text { Consolidation } \\
\text { consisted of } 4 \\
\text { additional cycles } \\
\text { of KPd, identical } \\
\text { to the induction } \\
\text { cycles. Patients } \\
\text { with } \\
\text { stable disease or } \\
\text { better received } \\
\text { Pomalidomide } \\
4 \text { mg w/o } \\
\text { Dexamethasone } \\
\text { in } 28 \text { days cycles } \\
\text { until } \\
\text { progression. }\end{array}$ & $\begin{array}{l}\text { Intravenous } \\
\text { carfilzomib } 20 \\
\text { mg/m (2) for all } \\
\text { treatment cycles, } \\
\text { and Cohort } 2 \text {, } \\
\text { scheduled to } \\
\text { receive } 20 \mathrm{mg} / \mathrm{m} \\
\text { (2) for cycle } 1 \\
\text { and then } 27 \mathrm{mg} / \\
\mathrm{m}(2) \text { for all } \\
\text { subsequent } \\
\text { cycles. }\end{array}$ & $\begin{array}{l}\text { Carfilzomib } 20 \\
\text { mg/m (2) } \\
\text { intravenously } \\
\text { on days } 1,2 \text {, } \\
8,9,15, \text { and } \\
16 \text { every } 28 \\
\text { days for up to } \\
12 \text { cycles. }\end{array}$ \\
\hline ORR & $65.20 \%$ & $58 \%$ & $50 \%$ & $87 \%$ & $\begin{array}{l}42.4 \% \text { in Cohort } 1 \\
\text { and } 52.2 \% \text { in } \\
\text { Cohort } 2\end{array}$ & $16.70 \%$ \\
\hline $\begin{array}{l}\text { Discontinuation } \\
\text { Rate }\end{array}$ & $\begin{array}{l}\mathrm{N}=4 \text { were lost to } \\
\text { follow-up and } \mathrm{N} \\
=10 \text { due to } \mathrm{PD}\end{array}$ & - & $\mathrm{N}=31$ & - & $\mathrm{N}=70$ & - \\
\hline $\begin{array}{l}\text { Median No. of } \\
\text { Previous Lines } \\
\text { of Therapies }\end{array}$ & $2(1-15)$ & - & $6(2-12)$ & 2 & $2(1-4)$ & - \\
\hline $\begin{array}{l}\text { Hematological } \\
\text { (Grade-3/4) } \\
\text { Toxicities }\end{array}$ & & & & $30 \%$ & & \\
\hline Thrombocytopenia & $\begin{array}{l}23.1 \% \text { (biweekly } \\
\text { regimen) } 20 \% \\
\text { (weekly regimen) }\end{array}$ & $13 \%$ & $\mathrm{~N}=7$ & NA & $13.20 \%$ & $50 \%$ \\
\hline
\end{tabular}


Table 4 continued

\begin{tabular}{|c|c|c|c|c|c|c|}
\hline Characteristics & Our Study & Bringhen et al [5] & Shah et al [6] & Sonneveld et al [7] & $\begin{array}{l}\text { PX-171-004 Trial } \\
\text { (Vij R et al) [13] }\end{array}$ & $\begin{array}{l}\text { PX-171-003-A0 } \\
\text { Trial } \\
\text { (Jagannath S } \\
\text { et al) [14] }\end{array}$ \\
\hline Neutropenia & $\begin{array}{l}28.2 \% \text { (biweekly } \\
\text { regimen) } 16.2 \% \\
\text { (weekly regimen) }\end{array}$ & $65 \%$ & $\mathrm{~N}=14$ & NA & $13.20 \%$ & - \\
\hline Anemia & $\begin{array}{l}20.5 \% \text { (biweekly } \\
\text { regimen) } 10 \% \\
\text { (weekly regimen) }\end{array}$ & - & $\mathrm{N}=6$ & NA & $41.90 \%$ & $73.90 \%$ \\
\hline PFS & $\begin{array}{l}\text { Median PFS of } \\
\text { entire cohort } 11.3 \\
\text { months (95\%C.I. } \\
8.3-14.2)\end{array}$ & Median9.5 months & $\begin{array}{l}\text { Median } 7.2 \\
\text { months ( } 95 \% \\
\text { C.I. 3-9 months) }\end{array}$ & $\begin{array}{l}\text { Median PFS was } \\
18 \text { months. }\end{array}$ & $\begin{array}{l}\text { Median PFS was } \\
8.3 \text { months }(95 \% \\
\text { C.I. 6-12.3) for } \\
\text { cohort } 1 \text { and not } \\
\text { reached for } \\
\text { cohort } 2\end{array}$ & - \\
\hline OS & $\begin{array}{l}\text { Median OS of } \\
\text { entire cohort was } \\
28 \text { months } \\
\text { (95\%C.I. } \\
\text { 20.4-35.5) }\end{array}$ & $\begin{array}{l}\text { Median OS was } \\
\text { not reached }\end{array}$ & $\begin{array}{l}\text { Median OS was } \\
20.6 \text { months } \\
\text { (95\% C.I. } \\
11.9-28.7 \\
\text { months) }\end{array}$ & $\begin{array}{l}\text { Median OS was } \\
\text { not reached }\end{array}$ & $\begin{array}{l}\text { Median OS was } \\
\text { not evaluable for } \\
\text { either cohort }\end{array}$ & - \\
\hline Mortality Rate & $\begin{array}{l}\mathrm{N}=3(12.50 \%) \mathrm{N} \\
\quad=1 \text { (biweekly } \\
\text { regimen) } \mathrm{N}=2 \\
\text { (weekly regimen) }\end{array}$ & - & $\mathrm{N}=2$ & $\mathrm{~N}=3$ & $\mathrm{~N}=4$ & - \\
\hline
\end{tabular}

et al [5] observed infections (11\%), vascular events $(9 \%)$ and cardiac events (4\%).

Similar results were observed in single centre Turkish study where most common haematological AEs were thrombocytopenia, anemia and neutropenia in $38 \%, 38 \%$ and $28.5 \%$ respectively whereas non-hematological events were fatigue $(71.4 \%)$, nausea $(66.7 \%)$ and dyspnoea $(28.5 \%)$ [11].

Another study from Israel [12] showed haematological toxicities such as anemia (76.3\%), thrombocytopenia (75.6\%) and non-hematological toxicities were cardiac related. Our results are comparable with these studies as well, with a non-caucasian population.

We encountered various infections in our study population. In biweekly and weekly regimen any grade infectious complications were seen in 15 (38.4\%) and 20 $(66.6 \%)$ patients respectivley. Common infections in biweekly regimen were bacterial $\{5(12.8 \%)\}$, viral $\{4(10.2 \%)\}$ and possible IFI $\{6(15.4 \%)\}$. Whereas in once weekly regimen, mainly bacterial infections were encounterd $\{4(13.3 \%)\}$ Shah JJ et al [6] and Sonneveld et al [7] reported infectious complication in $28.1 \%$ and $20 \%$ of patients respectively whereas $\mathrm{S}$. Bringhen et al [5] reported grade-3/4 infections in $n=5(11 \%)$.

In our study, median PFS was 11.3 months (95\%C.I. 8.3-14.2) whereas the estimated median OS was 28 months (95\%C.I. 20.4-35.5) for the entire cohort. . In the study by Shah et al [6] at a median follow-up of 26.3(range 1-37)months, median PFS and OS was 7.2 (95\% C.I. 3-9)months and 20.6 (95\% C.I. 11.9-28.7)months respectively. Comparison with other studies shown in Table 4.

In our study mortality was $2.5 \%$ versus $10 \%$ due to PD in biweekly and once weekly cohorts respectively. In contrast, mortality rate was $9.5 \%$ in Turkish study [11] and all were treatment related. Similarly, Shah et al [6] reported 2 deaths due to pneumonia and pulmonary embolism. Sonneveld et al [7] also observed 3 deaths due to cardiac toxicity and pneumonia whereas S. Bringhen et al [5] had no treatment related mortality in their study.

Although carfilzomib discontinuation had been observed in our weekly KPD cohort due to treatment related toxicities, no patient discontinued KPd therapy in biweekly regimen for the same.

In overall study period, 9patients received autoSCT and 21 patients received maintenance therapy \{carfilzomibdexamethasone: $(\mathrm{n}=5)$, pomalidomide-dexamethasone:(n $=9)$, pomalidomide: $(n=7)\}$. However, there was significant number of progression $(26 \%)$ and relapses $(34.7 \%)$ which questions the real effectiveness of this regimen in long term.

We acknowledge that our study had limitations like its retrospective nature, fewer patients and shorter duration of follow-up. In addition, cytogenetics for risk stratification was not available for all patients, hence we could not correlate response rate with R-ISS, especially in high risk 
myeloma. Possible IFI were not proven microbiologically and hence could be overestimated.

In conclusion, results of our study showed that KPd regimen is capable of serving as a bridge to AutoSCT in RRMM. However, this comes at the cost of significant side effects. Our experience demands further clinical trials on these novel agents with different dosing regimens, schedule and various combinations.

Acknowledgments We acknowledge all the staff and team members of department of Hematology and Bone Marrow Transplant unit, Rajiv Gandhi Cancer Institute and Research Centre, India in performing this study.

Funding This research did not receive any specific grant from funding agencies

Data Availability Retrospectively data collected in spreadsheet from hospital medical records

\section{Declarations}

Conflicts of interest All authors declare that they have no potential conflicts of interest to declare. No funding was received to assist with the preparation of this manuscript. No funding was received for conducting this study. No funds, grants, or other support was received. The authors have no relevant financial or non-financial interests to disclose. The authors have no conflicts of interest to declare that are relevant to the content of this article. All authors certify that they have no affiliations with or involvement in any organization or entity with any financial interest or non-financial interest in the subject matter or materials discussed in this manuscript. The authors have no financial or proprietary interests in any material discussed in this article.

Consent for Publication All authors gave their consent for publication of this study

Ethical Approval It is a retrospective study and this study was approved by Institutional Review Board for publication.

Human or Animal Rights This is a retrospective study which is conducted on already available data from medical record department of institution and not involving human participants and/or animals, therefore no formal consent is required to conduct the study. Institutional Review Board approval has received to conduct the study. Study was done with all ethical standards as per 1964 Helsinki Declaration and its later amendments or comparable ethical standards.

Informed Consent This study is a retrospective in nature and doesn't involve any human participant, hence no formal informed consent is required for the study. This study is a collection of data from medical record department hence no formal consent is required to conduct the study.

\section{References}

1. Weber DM, Chen C, Niesvizky R, Wang M, Belch A, Stadtmauer EA et al (2007) Lenalidomide plus dexamethasone for relapsed multiple myeloma in North America. $\mathrm{N}$ Engl $\mathrm{J}$ Med. 357(21):2133-42

2. Herndon TM, Deisseroth A, Kaminskas E, Kane RC, Koti KM, Rothmann MD et al (2013) U.S. food and drug administration approval: carfilzomib for the treatment of multiple myeloma. Clin Cancer Res 19(7):4559-63

3. Dimopoulos MA, Lacy MQ, Moreau P, Weisel KC, Song KW, Delforge $\mathrm{M}$ et al (2012) Pomalidomide in combination with lowdose dexamethasone: demonstrates a significant progression free survival and overall survival advantage, in relapsed/refractory MM: a phase 3, multicenter, randomized, open-label study. Blood. https://doi.org/10.1182/blood.V120.21.LBA-6.LBA-6

4. Miguel JS, Weisel K, Moreau P, Lacy M, Song K, Delforge M et al (2013) Pomalidomide plus low-dose dexamethasone versus high-dose dexamethasone alone for patients with relapsed and refractory multiple myeloma (MM-003): a randomised, open-label, phase 3 trial. Lancet Oncol. 14(11):1055-66

5. Bringhen S, Magarotto V, Liberati AM, Belotti A, Larocca A, Gilestro M et al (2016) A multicenter, open label phase I/II study of carfilzomib, pomalidomide and dexamethasone in relapsed and/or refractory multiple myeloma (MM) patients. Blood. 128(22):1145-1145

6. Shah JJ, Stadtmauer EA, Abonour R, Cohen AD, Bensinger WI, Gasparetto C et al (2015) Carfilzomib, pomalidomide, and dexamethasone for relapsed or refractory myeloma. Blood 126(20):2284-90

7. Sonneveld P, Zweegman S, Cavo M, Nasserinejad K, Troia R, Pour L et al (2018) Carfilzomib, pomalidomide and dexamethasone (KPd) in patients with multiple myeloma refractory to bortezomib and lenalidomide. The EMN011 Trial. Blood 132(Supplement 1):801-801

8. Costa LJ (2018) A phase $1 / 2$ trial of carfilzomib + high-dose melphalan conditioning for auto-HCT followed by carfilzomib maintenance for patients with relapsed multiple myeloma. Blood Marrow Transplant 24(7):1379-1385

9. Accesses on 29th of December, 2020. https://www.myeloma.org/ international-myeloma-working-group.

10. Accessed on 29th of December, 2020. National Cancer Institute Common Terminology Criteria for Adverse Events. https://ctep. cancer.gov/protocolDevelopment/electronic_applications/docs/ CTCAE_v5_Quick_Reference_8.5x11.pdf.

11. Uysal A, Akad SN (2018) Carfilzomib experience in relapsed/ refractory multiple myeloma: a single-center experience. Turk $\mathrm{J}$ Med Sci 48(1):80-3

12. Muchtar E, Gatt ME, Rouvio O, Ganzel C, Chubar E, Suriu C et al (2016) Efficacy and safety of salvage therapy using Carfilzomib for relapsed or refractory multiple myeloma patients: a multicentre retrospective observational study. $\mathrm{Br} \mathrm{J}$ Haematol. 172(1):89-96

13. Vij R, Wang M, Kaufman JL, Lonial S, Jakubowiak AJ, Stewart AK et al (2012) An open-label, single-arm, phase 2 (PX-171-004) study of single-agent carfilzomib in bortezomib-naive patients with relapsed and/or refractory multiple myeloma. Blood 119(24):5661-70

14. Jagannath S, Vij R, Stewart AK, Trudel S, Jakubowiak AJ, Reiman T, Somlo G, Bahlis N, Lonial S, Kunkel LA, Wong A, Orlowski RZ, Siegel DS (2012) An open-label single-arm pilot phase II study (PX-171-003-A0) of low-dose, single-agent carfilzomib in patients with relapsed and refractory multiple myeloma. Clin Lymphoma Myeloma Leuk. 12(5):310-8

Publisher's Note Springer Nature remains neutral with regard to jurisdictional claims in published maps and institutional affiliations. 